RASĀYAN J. Chem.

Vol. 13 | No. 1 |654 - 662| January - March | 2020 ISSN: 0974-1496 | e-ISSN: 0976-0083 | CODEN: RJCABP

\title{
SYNTHESIS OF SOME CHALCONE DERIVATIVES, IN VITRO AND IN SILICO TOXICITY EVALUATION
}

\author{
A. N. Kristanti", H. Suwito, N.S. Aminah, K.U. Haq, H. D. Hardiyanti, \\ H. Anggraeni, N. Faiza, R.S. Anto and S. Muharromah \\ Department of Chemistry, Faculty of Science and Technology, Universitas Airlangga, \\ Surabaya-60115, Indonesia \\ *E-mail: alfinda-n-k@fst.unair.ac.id
}

\begin{abstract}
Chalcone can be synthesized using some methods, but conventional Claisen-Schmidt condensation is still the best method. The objevtives of this study were to synthesize some chalcone derivatives using conventional ClaisenSchmidt condensation by reacting 2-hydroxyacetophenone and some substituted benzaldehydes using $\mathrm{NaOH} 40 \%$, followed by evaluating their cytotoxicity in vitro against HeLa cancer cells line using MTT method and analyzing molecular docking on p53 and MDM2 interaction. Cytotoxicity test exhibited that 2,5-dimethoxy-2'hydroxychalcone and 4-chloro-2'-hydroxychalcone gave very low $\mathrm{IC}_{50}$, but both did not show potential apoptosis activity, while in docking analysis 4-chloro-2'-hydroxychalcone showing the best results.

Keywords: 2-Hydroxychalcone Derivatives, Claisen-Schmidt Condensation, HeLa Cell, Apoptosis, MDM2 Protein.

(C) RASĀYAN. All rights reserved
\end{abstract}

\section{INTRODUCTION}

Chalcones belong to one of secondary metabolites produced by plants showing various pharmacological activities, such as antimicrobial and antifungal ${ }^{+}$, anti-tumor and anti-angiogenic ${ }^{2}$, antiinflamation, cytotoxic and antioxidant ${ }^{3}$, anticancer ${ }^{4}$, antileishmanial ${ }^{5}$, antibacterial ${ }^{6}$, antimalarial ${ }^{7}$, and antidiabetic. ${ }^{(1-8)}$ Chalcone can be synthesized using some organic reactions, such as Claisen-Schmidt, Suzuki, Wittig, Friedel-Craft acylation of cinnamoyl chloride and phenyl cinammic acid photo-Fries rearrangement. Various catalysts and reagents have been used in chalcone synthesis, for example $\mathrm{SOCl}_{2}$, natural phosphat, lithium nitrate, amino grafted zeolite, $\mathrm{ZnO}, \mathrm{Na}_{2} \mathrm{CO}_{3}$, PEG-400, silica sulfate, $\mathrm{ZrCl}_{4}$, and ionic liquid. ${ }^{9}$ To date, conventional Claisen-Schmidt condensation is still the best method to synthesize chalcone. This method is carried out using alkali solution as catalyst, microwave or ultrasound irradiation. Almost $75 \%$ of chalcone synthesis is performed using alkali solution. ${ }^{10}$ Synthesis using microwave irradiation takes a shorter time which can be more effective, faster, and energy efficient in addition ${ }^{11}$. Nevertheless, some researcher found that in this methode difficult to control the reaction, so that many by-products are formed. As a consequence, further separation is necessary. ${ }^{12}$ Chalcones synthesis was observed under ultrasound irradiation for a notable enhancing effect on the time of reaction and yield. ${ }^{13}$

In this study, some chalcone derivatives (compound 1-8) were succesfully synthesized using classical Claisen-Schmidt condensation by reacting 2-hydroxyacetophenone and some substituted benzaldehydes using $\mathrm{NaOH} 40 \%$ (Fig.-1 and Table-1).<smiles>CC(=O)c1ccccc1O</smiles><smiles>[R]c1ccc(C(=O)[OH2+])cc1</smiles><smiles>[R]c1ccc(/C=C/C(=O)c2ccccc2O)cc1</smiles>

Fig.-1: Synthesis Reaction for Chalcone Derivatives Using Claisen Schmidt Condensation

Rasayan J. Chem., 13(1), 654-662(2020)

http://dx.doi.org/10.31788/RJC.2020.1315534

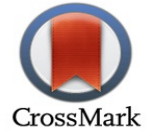


RASĀYAN J. Chem.

Vol. 13 | No. 1 |654 - 662 | January - March | 2020

Table-1: The Synthezised Chalcones

\begin{tabular}{c|c|c}
\hline Compounds & $\mathrm{R}$ & Compound's Name \\
\hline 1 & 2-OMe & 2-methoxy-2'-hydroxychalcone \\
\hline 2 & 3-OMe & 3-methoxy-2'-hydroxychalcone \\
\hline 3 & 4-OMe & 4-methoxy-2'-hydroxychalcone \\
\hline 4 & 2,4-diOMe & 2,4-dimethoxy-2'-hydroxychalcone \\
\hline 5 & $2,5-d i O M e$ & 2,5-dimethoxy-2'-hydroxychalcone \\
\hline 6 & 4-(N,N-diCH & 4-N,N-dimethyl-2'-hydroxychalcone \\
\hline 7 & $4-\mathrm{F}$ & 4-fluro-2'-hydroxychalcone \\
\hline 8 & 4-Cl & 4-chloro-2'-hydroxychalcone \\
\hline
\end{tabular}

In cancer cases, overexpression of oncoprotein MDM2, as well as inactivation of p53 gene is observed. As a result, the tumor supressor protein p53 was inhibited by the oncoprotein MDM2 that was bound to the transactivation domain of $\mathrm{p} 53 .{ }^{14,15}$ Therefore reactivation of $\mathrm{p} 53$ activity through inhibition of MDM2/p53 interaction is a promising mechanism to find anticancer agents. As reported by Stoll et al, some chalcone derivatives were found to show anticancer activity through this mechanism with moderate activity. ${ }^{16}$ Based on this report, we studied the cytotoxicity of some hydroxychalcone derivatives attaching substituents that withdraw and donate electrons.

Cytotoxic activity against HeLa cancer cells of the prepared chalcones were determined by MTT assay, while their apoptosis mechanism test was determined using flow cytometry experiment. Furthermore, molecular interaction between MDM2 and the prepared chalcones was studied by molecular docking experiment

\section{Material and Methods}

\section{EXPERIMENITAL}

All chemicals were provided from E.Merck in Darmstadt, Germany and Sigma-Aldrich in St. Louis, the United States and used directly without prior purification. Melting-point determination was conducted using Fisher-Johns melting point apparatus (Fisher Scientific Serial 40400075) and it was uncorrected. Each type of spectrum was recorded using different instruments : UV-Vis spectrophotometer Shimadzu UV-1800 was used to record UV-Vis spectra, Fourier Transform Infrared spectroscopy (FTIR) Shimadzu IRTracer-100 was used to record IR spectra, whereas Nuclear Magnetic Resonance (NMR) JEOL JNMECS 400 was used to record ${ }^{1} \mathrm{H}$ and ${ }^{13} \mathrm{C}$ NMR spectra.

\section{General Procedure}

\section{The Synthesis Of Chalcones (1-8)}

This procedure refered to Suwito et al. ${ }^{17}$ A solution of 2'-hydroxyacetophenone (6 mmol) and benzaldehyde derivatives $(6 \mathrm{mmol})$ in $30 \mathrm{~mL}$ of ethanol was stirred and refluxed at $5-10^{\circ} \mathrm{C}$ until the mixture formed a homogenous solution. Then $6 \mathrm{~mL}$ of $\mathrm{NaOH} 40 \%$ solution was added dropwise and the reaction mixture was kept under $10^{\circ} \mathrm{C}$ under stirring for 1 hour. The stirring was then kept on going at room temperature for one night. The mixture of reaction was transferred into crush ice and under stirring, then $15 \mathrm{~mL}$ of $\mathrm{H}_{2} \mathrm{SO}_{4} 4 \mathrm{~N}$ solution was added dropwise. The precipitate was filtered off, dried and recrystallized using ethanol yielding 40\%-90\%.

\section{2-methoxy-2'-hydroxychalcone (1)}

UV-Vis $(\mathrm{EtOH}) \lambda_{\max }(\mathrm{nm}): 366(0.521) ; 308$ (0.353); $250(0.249)$. FTIR (KBr) $\tilde{v}\left(\mathrm{~cm}^{-1}\right): 3300(\mathrm{OH}$ phenolic); 3047 (C-H aromatic); $2964\left(\mathrm{C}-\mathrm{H} \mathrm{sp}{ }^{3}\right) ; 1691(\mathrm{C}=\mathrm{O}$ conjugated); 1643 (C=C conjugated); 1581 $\left(\mathrm{C}=\mathrm{C}\right.$ aromatic); 1251 (C-O phenolic); 1205 (C-O methoxy). ${ }^{1} \mathrm{H}-\mathrm{NMR}\left(400 \mathrm{MHz}, \mathrm{CDCl}_{3}\right) \delta(\mathrm{ppm})$ : $12.95(1 \mathrm{H}, \mathrm{s}, \mathrm{OH}) ; 8.22\left(1 \mathrm{H}, \mathrm{d}, J=15.6 \mathrm{~Hz}, \mathrm{H}_{\beta}\right) ; 7.92(1 \mathrm{H}, \mathrm{dd}, J=1.7 \& 8.1 \mathrm{~Hz}, \mathrm{H}-6$ '); $7.78(1 \mathrm{H}, \mathrm{d}, J=15.6$ $\left.\mathrm{Hz}, \mathrm{H}_{\alpha}\right) ; 7.64\left(1 \mathrm{H}, \mathrm{dd}, J=8.6 \& 1.7 \mathrm{~Hz}, \mathrm{H}-3^{\prime}\right) ; 7.48\left(1 \mathrm{H}, \mathrm{td}, J=8.6 ; 7.2 ; 1.7 \mathrm{~Hz}, \mathrm{H}-4^{\prime}\right) ; 7.40$ (1H, td, $J=8.1$; 7.2; $\left.1.7 \mathrm{~Hz}, \mathrm{H}-5^{\prime}\right) ; 7.04-6.91$ (4H, m, H-3, H-4, H-5, H-6); 3.93 (3H, s, $\left.\mathrm{OCH}_{3}\right) .{ }^{13} \mathrm{C}-\mathrm{NMR}(100.5 \mathrm{MHz}$, $\left.\mathrm{CDCl}_{3}\right) \delta(\mathrm{ppm}): 194.4(\mathrm{C}, \mathrm{C}=\mathrm{O}) ; 163.7\left(\mathrm{C}, \mathrm{C}-2^{\prime}\right) ; 159.1(\mathrm{C}, \mathrm{C}-2) ; 141.2(\mathrm{CH}, \mathrm{C} \beta) ; 136.3\left(\mathrm{CH}, \mathrm{C}-4^{\prime}\right)$; $132.3(\mathrm{CH}, \mathrm{C}-4) ; 129.8\left(\mathrm{CH}, \mathrm{C}-6{ }^{\prime}\right) ; 129.8(\mathrm{CH}, \mathrm{C}-6) ; 123.7(\mathrm{C}, \mathrm{C} 1) ; 120.9(\mathrm{CH} ; \mathrm{C \alpha}) ; 120.8(\mathrm{CH}, \mathrm{C}-5)$; $120.3\left(\mathrm{C}, \mathrm{C}-1^{\prime}\right) ; 118.9\left(\mathrm{CH}, \mathrm{C}-5^{\prime}\right) ; 118.6\left(\mathrm{CH}, \mathrm{C}-3^{\prime}\right) ; 114.4(\mathrm{CH}, \mathrm{C}-3) ; 55.7\left(\mathrm{CH}_{3}, \mathrm{OCH}_{3}\right)$. HRMS : $277.08336(\mathrm{M}+\mathrm{Na})$ suitable for molecular formula of $\mathrm{C}_{16} \mathrm{H}_{14} \mathrm{O}_{3}$ 
RASĀYAN J. Chem.

Vol. 13 | No. 1 |654 - 662| January - March | 2020

\section{3-methoxy-2'-hydroxychalcone (2)}

UV-Vis (EtOH) $\lambda_{\max }(\mathrm{nm}): 312$ (0.761); 254 (0.419). FTIR (KBr) $\tilde{v}\left(\mathrm{~cm}^{-1}\right): 3300$ (OH phenolic); 3012 (C-H aromatic); $2966\left(\mathrm{C}-\mathrm{H} \mathrm{sp}^{3}\right) ; 1637(\mathrm{C}=\mathrm{O}$ conjugated); $1602(\mathrm{C}=\mathrm{C}$ conjugated $) ; 1577(\mathrm{C}=\mathrm{C}$ aromatic); 1259 (C-O phenolic); 1209 (C-O methoxy). ${ }^{1} \mathrm{H}-\mathrm{NMR}\left(400 \mathrm{MHz}, \mathrm{CDCl}_{3}\right) \delta(\mathrm{ppm}): 12.81(1 \mathrm{H}, \mathrm{s}, \mathrm{OH})$; $7.87\left(1 \mathrm{H}, \mathrm{d}, J=15.5 \mathrm{~Hz}, \mathrm{H}_{\beta}\right) ; 7.91\left(1 \mathrm{H}, \mathrm{dd}, J=1.3 \& 8.0 \mathrm{~Hz}, \mathrm{H}-6{ }^{\prime}\right) ; 7.62\left(1 \mathrm{H}, \mathrm{d}, J=15.5 \mathrm{~Hz}, \mathrm{H}_{\alpha}\right) ; 7.64(1 \mathrm{H}$, $\left.\mathrm{dd}, J=8.6 \& 1.7 \mathrm{~Hz}, \mathrm{H}-3^{\prime}\right) ; 7.49$ (1H, td, $\left.J=8.4 ; 7.1 ; 1.3 \mathrm{~Hz}, \mathrm{H}-4^{\prime}\right) ; 7.40\left(1 \mathrm{H}, \mathrm{td}, J=8.1 ; 7.2 ; 1.7 \mathrm{~Hz}, \mathrm{H}-5^{\prime}\right)$; $7.34(1 \mathrm{H}, \mathrm{m}, \mathrm{H}-5) ; 7.25(1 \mathrm{H}, \mathrm{m}, \mathrm{H}-4) ; 7.16(1 \mathrm{H}, \mathrm{m}, \mathrm{H}-2) ; 7.05-6.91(3 \mathrm{H}, \mathrm{m}, \mathrm{H}-3$ ', H-5', H-6); 3.86 (3H, s, $\left.\mathrm{OCH}_{3}\right) .{ }^{13} \mathrm{C}-\mathrm{NMR}\left(100.5 \mathrm{MHz}, \mathrm{CDCl}_{3}\right) \delta(\mathrm{ppm}): 193.8(\mathrm{C}, \mathrm{C}=\mathrm{O}) ; 163.7\left(\mathrm{C}, \mathrm{C}-2{ }^{\prime}\right) ; 160.1(\mathrm{C}, \mathrm{C}-3)$;

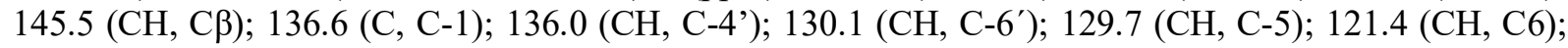
120.5 (CH; $\mathrm{C \alpha}) ; 120.8$ (CH, C-5); 120.1 (C, C-1'); 119.0 (CH, C-5'); 118.7 (CH, C-4); 116.7 (CH, C-3'); $113.8(\mathrm{CH}, \mathrm{C}-2)$; $55.5\left(\mathrm{CH}_{3}, \mathrm{OCH}_{3}\right)$. HRMS : $277.08301(\mathrm{M}+\mathrm{Na})$ suitable for molecular formula of $\mathrm{C}_{16} \mathrm{H}_{14} \mathrm{O}_{3}$

\section{4- methoxy -2'- hydroxychalcone (3)}

UV-Vis $(\mathrm{EtOH}) \lambda_{\max }(\mathrm{nm}): 362(0.888)$. FTIR $(\mathrm{KBr}) \tilde{v}\left(\mathrm{~cm}^{-1}\right): 3300(\mathrm{OH}$ phenolic); $3024(\mathrm{C}-\mathrm{H}$ aromatic); $2970\left(\mathrm{C}-\mathrm{H} \mathrm{sp}{ }^{3}\right) ; 1637(\mathrm{C}=\mathrm{O}$ conjugated $) ; 1606(\mathrm{C}=\mathrm{C}$ conjugated $) ; 1562(\mathrm{C}=\mathrm{C}$ aromatic $) ; 1233$ (C-O phenolic); 1211 (C-O methoxy). ${ }^{1} \mathrm{H}-\mathrm{NMR}\left(400 \mathrm{MHz}, \mathrm{CDCl}_{3}\right) \delta(\mathrm{ppm}): 12.95(1 \mathrm{H}, \mathrm{s}, \mathrm{OH}) ; 7.87-$

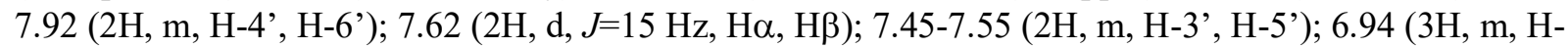
2, H-3, H-5). ${ }^{13} \mathrm{C}-\mathrm{NMR}\left(100.5 \mathrm{MHz}, \mathrm{CDCl}_{3}\right) \delta(\mathrm{ppm}): 194.8(\mathrm{C}, \mathrm{C}=\mathrm{O}) ; 163.6(\mathrm{C}, \mathrm{C}-2$ '); 162.1 (C-C-4); 145.5 (CH, Cß); 136.3 (CH, C-4'); 130.7 (2CH, C-2, C-6); 129.9 (CH, C-6'); 127.4 (C, C-1); 120.2 (C, C1'); $118.9(\mathrm{CH}, \mathrm{C} \alpha) ; 118.7\left(\mathrm{CH}, \mathrm{C}-5\right.$ '); $117.6\left(\mathrm{CH}, \mathrm{C}-3\right.$ '); 114.6 (2CH, C-3, C-5); $55.55\left(\mathrm{OCH}_{3}\right)$. HRMS : $277.08526(\mathrm{M}+\mathrm{Na})$ suitable for molecular formula of $\mathrm{C}_{16} \mathrm{H}_{14} \mathrm{O}_{3}$

\section{2,4- dimethoxy-2'-hydroxychalcone (4)}

UV-Vis (EtOH) $\lambda_{\max }(\mathrm{nm}): 385$ (0.583); 254 (0.288), FTIR (KBr) $\tilde{v}\left(\mathrm{~cm}^{-1}\right): 3300$ (OH phenolic); 3072 (C-H aromatic); $2950\left(\mathrm{C}-\mathrm{H} \mathrm{sp}^{3}\right) ; 1635(\mathrm{C}=\mathrm{O}$ conjugated $), 1608(\mathrm{C}=\mathrm{C}$ conjugated $) ; 1550(\mathrm{C}=\mathrm{C}$ aromatic); 1228 (C-O phenolic), 1228 (C-O methoxy). ${ }^{1} \mathrm{H}-\mathrm{NMR}\left(400 \mathrm{MHz}, \mathrm{CDCl}_{3}\right) \delta_{\mathrm{H}}(\mathrm{ppm}): 13.10$ (s, OH); 8.15 $(\mathrm{d}, 1 \mathrm{H}, J=15.5 \mathrm{~Hz}) ; 7.90(\mathrm{~d}, 1 \mathrm{H}, J=8.0 \mathrm{~Hz}) ; 7.68(\mathrm{~d}, 1 \mathrm{H}, J=15.4 \mathrm{~Hz}) ; 7.57(\mathrm{~d}, 1 \mathrm{H}, J=8.6 \mathrm{~Hz}) ; 7.46(\mathrm{t}$, $1 \mathrm{H}, J=7.8 \mathrm{~Hz}) ; 3.85$ (s, 3H); $7.00(\mathrm{~d}, 1 \mathrm{H}, J=8.4 \mathrm{~Hz}) ; 6.91(\mathrm{t}, 1 \mathrm{H}, J=7.6 \mathrm{~Hz}) ; 6.53(\mathrm{dd}, 1 \mathrm{H}, J=8.6 ; 2.0$ $\mathrm{Hz}) ; 6.47(\mathrm{~d}, 1 \mathrm{H}, J=1.8 \mathrm{~Hz}) ; 3.91(\mathrm{~s}, 3 \mathrm{H}) .{ }^{13} \mathrm{C}-\mathrm{NMR}\left(100.5 \mathrm{MHz}, \mathrm{CDCl}_{3}\right) \delta_{\mathrm{c}}(\mathrm{ppm}): 194.4(\mathrm{C}, \mathrm{C}=\mathrm{O})$; 163.6 (C, C-2'); 163.5 (C, C-4); 160.8 (C, C-2); 141.4 (CH, C- $\beta$ ); 136.0 (CH, C-4'); 131.6 (CH, C-6); 129.7 (CH, C-6'); 120.4 (C, C-1'); 118.8 (CH, C-5'); $118.6(\mathrm{CH}, \mathrm{C}-\alpha)$; 118.1 (CH, C-3'); 116.9 (C, C-1); $105.7(\mathrm{CH}, \mathrm{C}-5)$; $98.5(\mathrm{CH}, \mathrm{C}-\mathrm{C}-3) ; 55.7\left(\mathrm{OCH}_{3}\right) ; 55.6\left(\mathrm{OCH}_{3}\right)$. HRMS : $307.09543(\mathrm{M}+\mathrm{Na})$ suitable for molecular formula of $\mathrm{C}_{17} \mathrm{H}_{16} \mathrm{O}_{4}$

\section{2,5- dimethoxy -2'-hydroxychalcone (5)}

UV-Vis $(\mathrm{EtOH}) \lambda_{\max }(\mathrm{nm}): 310(0.789)$. FTIR $(\mathrm{KBr}) \tilde{v}\left(\mathrm{~cm}^{-1}\right): 3300(\mathrm{OH}$ phenolic); $3068(\mathrm{C}-\mathrm{H}$ aromatic); $2962\left(\mathrm{C}-\mathrm{H}\right.$ sp$\left.^{3}\right) ; 1639(\mathrm{C}=\mathrm{O}$ conjugated $) ; 1610(\mathrm{C}=\mathrm{C}$ conjugated $) ; 1500(\mathrm{C}=\mathrm{C}$ aromatic $) ; 1222$ (C-O phenolic); 1220 (C-O methoxy);. ${ }^{1} \mathrm{H}-\mathrm{NMR}\left(400 \mathrm{MHz}, \mathrm{CDCl}_{3}\right) \delta_{\mathrm{H}}(\mathrm{ppm}): 12.92(1 \mathrm{H}, \mathrm{s}, \mathrm{OH}) ; 8.18$ $(1 \mathrm{H}, \mathrm{d}, J=15.6 \mathrm{~Hz}, \mathrm{H} \beta) ; 7.91(1 \mathrm{H}, \mathrm{dd}, J=1.8 \& 7.5 \mathrm{~Hz} ; \mathrm{H}-6$ '); $7.74(1 \mathrm{H}, \mathrm{d}, \mathrm{H}-4$ ', H-6'); 7.62 (2H, d, $J=15.6 \mathrm{~Hz}, \mathrm{H \alpha}) ; 7.48\left(1 \mathrm{H}, \mathrm{m}, \mathrm{H}-4{ }^{\prime}\right) ; 7.15(1 \mathrm{H}, \mathrm{d}, J=3 \mathrm{~Hz}, \mathrm{H}-6) ; 7.01(1 \mathrm{H}, \mathrm{d}, J=7.9 \mathrm{~Hz}, \mathrm{H}-3) ; 6.88-6.97$ $\left(2 \mathrm{H}, \mathrm{m}, \mathrm{H}-3\right.$ ', H-5'); $6.94(1 \mathrm{H}, \mathrm{dd}, J=3.0 \& 7.9 \mathrm{~Hz}, \mathrm{H}-4) ; 3.88\left(3 \mathrm{H}, \mathrm{s}, \mathrm{OCH}_{3}\right) ; 3.82\left(3 \mathrm{H}, \mathrm{s}, \mathrm{OCH}_{3}\right) .{ }^{13} \mathrm{C}-$ NMR (100.5 MHz, $\left.\mathrm{CDCl}_{3}\right) \delta_{\mathrm{c}}(\mathrm{ppm}): 194.3(\mathrm{C}, \mathrm{C}=\mathrm{O}) ; 163.6$ (C, C-2'); 153.7 (C-C-2); 153.6 (CH, C-5);

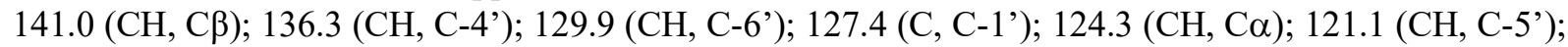
120.3 (C, C-1); 118.9 (CH, C-1'); 118.7 (CH, C-3); 117.8 (CH, C-3'); 114.3 (CH, C-4); 112.6 (CH, C-6); $56.2\left(\mathrm{OCH}_{3}\right) ; 56.0\left(\mathrm{OCH}_{3}\right)$. HRMS : $307.09460(\mathrm{M}+\mathrm{Na})$ suitable for molecular formula of $\mathrm{C}_{17} \mathrm{H}_{16} \mathrm{O}_{4}$

\section{4-N,N-dimethyl-2'-hydroxychalcone (6)}

UV-Vis (EtOH) $\lambda_{\text {maks }}(\mathrm{nm}): 437$ (57.043); 275 (24.259). FTIR (KBr) $\tilde{v}\left(\mathrm{~cm}^{-1}\right): 3300$ (OH phenolic); 2940 $\left(\mathrm{C}-\mathrm{H} \mathrm{sp}^{3}\right) ; 1620(\mathrm{C}=\mathrm{O}$ conjugated $), 1598(\mathrm{C}=\mathrm{C}$ conjugated $) ; 1580(\mathrm{C}=\mathrm{C}$ aromatic); $1342(\mathrm{C}-\mathrm{N}) ; 1207(\mathrm{C}-$ O phenolic). ${ }^{1} \mathrm{H}-\mathrm{NMR}\left(400 \mathrm{MHz}, \mathrm{CDCl}_{3}\right) \delta_{\mathrm{H}}(\mathrm{ppm}): 13.22(\mathrm{~s}, \mathrm{OH}) ; 7.91(\mathrm{~d}, 1 \mathrm{H}, J=15.4 \mathrm{~Hz}) ; 7.90$ (dd, $1 \mathrm{H}, J=7.2 \& 1.2 \mathrm{~Hz}) ; 7.56(\mathrm{~d}, 2 \mathrm{H}, J=8.9 \mathrm{~Hz}) ; 7.45(\mathrm{td}, 1 \mathrm{H}, J=8.4 ; 7.2 ; 1.6 \mathrm{~Hz}) ; 7.44(\mathrm{~d}, 1 \mathrm{H}, J=15.4$ 
RASĀYAN J. Chem.

Vol. 13 | No. 1 |654 - 662| January - March | 2020

$\mathrm{Hz}) ; 7.00(\mathrm{dd}, 1 \mathrm{H}, J=9.4 ; \& 1.2 \mathrm{~Hz}) ; 6.91(\mathrm{td}, 1 \mathrm{H}, J=8.4 ; 7.2 ; 1.2 \mathrm{~Hz}) ; 6.68(\mathrm{~d}, 2 \mathrm{H}, J=8.9 \mathrm{~Hz}) ; 3.04$ (s, 6H). ${ }^{13} \mathrm{C}-\mathrm{NMR}\left(100.5 \mathrm{MHz}, \mathrm{CDCl}_{3}\right) \delta_{\mathrm{c}}(\mathrm{ppm}): 193.6(\mathrm{C}, \mathrm{C}=\mathrm{O}) ; 163.6(\mathrm{C}, \mathrm{C}-2) ; 152.4(\mathrm{C}, \mathrm{C}-4) ; 146.7$ $(\mathrm{CH}, \mathrm{C}-\beta) ; 135.8$ (CH, C-4'); 131.0 (2CH, C-2 \& C-6); 129.5 (CH, C-6'); 122.4 (C, C-1); 120.5 (C, C1'); $118.7(\mathrm{CH}, \mathrm{C}-\alpha)$; $118.6\left(\mathrm{CH}, \mathrm{C}-5\right.$ '); $114.3\left(\mathrm{CH}, \mathrm{C}-3\right.$ '); 111.9 (2CH, C-3 \& C-5); $40.2\left(2 \mathrm{CH}_{3}\right)$. HRMS : $290.11583(\mathrm{M}+\mathrm{Na})$ suitable for molecular formula of $\mathrm{C}_{17} \mathrm{H}_{17} \mathrm{NO}_{2}$

\section{4-fluoro-2'-hydroxychalcone (7)}

$\mathrm{UV}(\mathrm{EtOH}) \lambda_{\text {maks }}(\mathrm{nm}),(\log \varepsilon): 317.9$ (4.1); 223.9 (4.0). FTIR (KBr) $\tilde{v}\left(\mathrm{~cm}^{-1}\right): 3300$ (OH phenolic); 3035 (C-H aromatic); $1638(\mathrm{C}=\mathrm{O}$ conjugated), $1580(\mathrm{C}=\mathrm{C}$ conjugated); $1493(\mathrm{C}=\mathrm{C}$ aromatic); 1209 (C-O phenolic). ${ }^{1} \mathrm{H}-\mathrm{NMR}\left(400 \mathrm{MHz}, \mathrm{CDCl}_{3}\right) \delta_{\mathrm{H}}(\mathrm{ppm}): 12.80(\mathrm{~s}, \mathrm{OH}) ; 7.91$ (dd, $\left.1 \mathrm{H}, J=8.1 \& 1.7 \mathrm{~Hz}\right) ; 7.88(\mathrm{~d}$, $1 \mathrm{H}, J=15.6 \mathrm{~Hz}) ; 7.66\left(\mathrm{dd}, 2 \mathrm{H}, J=8.7 \mathrm{~Hz},{ }^{4} J_{\mathrm{H}-\mathrm{F}}=5.4 \mathrm{~Hz}\right) ; 7.58(\mathrm{~d}, 1 \mathrm{H}, J=15.6 \mathrm{~Hz}) ; 7.51(\mathrm{td}, 1 \mathrm{H}, J=$ $8.5 ; 7.2 ; 1.7 \mathrm{~Hz}) ; 7.13\left(\mathrm{t}, 2 \mathrm{H}, J=8.7 \mathrm{~Hz},{ }^{3} J_{\mathrm{H}-\mathrm{F}}=8.7 \mathrm{~Hz}\right) ; 7.03(\mathrm{dd}, 1 \mathrm{H}, J=8.5 \& 1.2 \mathrm{~Hz}) ; 6.95(\mathrm{td}, 1 \mathrm{H}, J$ $=8.1 ; 7.2 ; 1.2 \mathrm{~Hz}) .{ }^{13} \mathrm{C}-\mathrm{NMR}\left(100.53 \mathrm{MHz}, \mathrm{CDCl}_{3}\right) \delta_{\mathrm{C}}(\mathrm{ppm}): 193.7(\mathrm{C}, \mathrm{C}=\mathrm{O}) ; 164.4\left(\mathrm{~d},{ }^{1} J_{\mathrm{C}-\mathrm{F}}=252.6 \mathrm{~Hz}\right.$, C-4); 163.7 (C, C-2'); $144.3(\mathrm{CH}, \mathrm{C}-\beta) ; 136.6\left(\mathrm{CH}, \mathrm{C}-4^{\prime}\right) ; 131.0\left(\mathrm{~d},{ }^{4} J_{\mathrm{C}-\mathrm{F}}=3.4 \mathrm{~Hz}, \mathrm{C}-1\right) ; 130.8\left(\mathrm{~d},{ }^{3} J_{\mathrm{C}-\mathrm{F}}=\right.$ 8.6 Hz, C-2 \& C-6); 129.7 (CH-C-6'); 120.1 (C, C-1'); 119.9 (CH, C- $\alpha$ ); 119.0 (CH, C-5'); 118.8 (CH, $\mathrm{C}-3$ '); $116.4\left(\mathrm{~d},{ }^{2} J_{\mathrm{C}-\mathrm{F}}=22.0 \mathrm{~Hz}, \mathrm{C}-3\right.$ \& $\left.\mathrm{C}-5\right)$. HRMS : $265.06225(\mathrm{M}+\mathrm{Na})$ suitable for molecular formula of $\mathrm{C}_{15} \mathrm{H}_{11} \mathrm{FO}_{2}$

\section{4-chloro-2'-hydroxychalcone (8)}

$\mathrm{UV}(\mathrm{EtOH}) \lambda_{\text {maks }}(\mathrm{nm}),(\log \varepsilon): 320.0$ (4.2); 221.0 (4.2). FTIR (KBr) $\tilde{v}\left(\mathrm{~cm}^{-1}\right): 3300$ (OH phenolic); 3059 $(\mathrm{C}-\mathrm{H}$ aromatic); $1641(\mathrm{C}=\mathrm{O}$ conjugated $) ; 1578(\mathrm{C}=\mathrm{C}$ conjugated $) ; 1487(\mathrm{C}=\mathrm{C}$ aromatic $) ; 1206(\mathrm{C}-\mathrm{O}$ phenolic). ${ }^{1} \mathrm{H}-\mathrm{NMR}\left(400 \mathrm{MHz}, \mathrm{CDCl}_{3}\right) \delta_{\mathrm{H}}(\mathrm{ppm}): 12.76(\mathrm{~s}, \mathrm{OH}) ; 7.91$ (dd, $\left.1 \mathrm{H}, J=8.0 \& 1.8 \mathrm{~Hz}\right) ; 7.86(\mathrm{~d}$, $1 \mathrm{H}, J=15.4 \mathrm{~Hz}) ; 7.62(\mathrm{~d}, 1 \mathrm{H}, J=15.4 \mathrm{~Hz}) ; 7.59(\mathrm{~d}, 2 \mathrm{H}, J=8.5 \mathrm{~Hz}) ; 7.51(\mathrm{td}, 1 \mathrm{H}, J=8.6 ; 7.2 ; 1.8 \mathrm{~Hz})$; $7.41(\mathrm{~d}, 2 \mathrm{H}, J=8.5 \mathrm{~Hz}) ; 7.03(\mathrm{dd}, 1 \mathrm{H}, J=8.6 \& 1.3 \mathrm{~Hz}) ; 6.95(\mathrm{td}, 1 \mathrm{H}, J=8.0 ; 7.2 ; 1.3 \mathrm{~Hz}) .{ }^{13} \mathrm{C}-\mathrm{NMR}$ $\left(100.53 \mathrm{MHz}, \mathrm{CDCl}_{3}\right) \delta_{\mathrm{C}}(\mathrm{ppm}): 193.6(\mathrm{C}, \mathrm{C}=\mathrm{O}) ; 163.7$ (C, C-2'); $144.1(\mathrm{CH}, \mathrm{C}-\beta) ; 137.0(\mathrm{C}, \mathrm{C}-1) ; 136.7$ (CH, C-4'); 133.2 (C, C-4); 129.9 (CH, C-6'); 129.7 (2CH, C-2 \& C-6); 129.5 (2CH, C-3 \& C-5); 120.7 $(\mathrm{CH}, \mathrm{C}-\alpha) ; 120.0\left(\mathrm{C}, \mathrm{C}-1^{\prime}\right) ; 119.0\left(\mathrm{CH}, 5^{\prime}\right) ; 118.8\left(\mathrm{CH}, 3^{\prime}\right)$. HRMS : $281.03307(\mathrm{M}+\mathrm{Na})$ suitable for molecular formula of $\mathrm{C}_{15} \mathrm{H}_{11} \mathrm{ClO}_{2}$

\section{Cytotoxic Assay}

HeLa cells were cultured in a 96-well plate at $213.4 \times 10^{4}$ cell/well density. The cells were then treated with $100 \mu \mathrm{L}$ of the prepared compounds with various concentrations $(1.5625 ; 3.125 ; 6.25 ; 12.5 ; 25 ; 50$; dan and $100 \mu \mathrm{g} / \mathrm{mL}$ ) for 24 hours. MTT (3-[4,5-dimethylthiazol-2-yl]-2,5-diphenyltetrazolium bromide solution (50 mg MTT in $10 \mathrm{~mL}$ of PBS) $100 \mu \mathrm{L}$ was added, followed by incubation in $\mathrm{CO}_{2}$ incubator for 4 hours. The amount of viable cells was visualized by the formation of purple color due to formation of formazan crystals. The formed formazan that was proportionate to the total of viable cells was calculated using spectrophotometer at $560 \mathrm{~nm}$. The number of viable cells was calculated using following formula

$$
\% \text { Viable Cells }=\frac{\text { Treatment Absorbance }- \text { Control Medium Absorbance }}{\text { Negative Control Absorbance }- \text { Control Medium Absorbance }} \times 100 \%
$$

$\mathrm{IC}_{50}$ was obtained after statistical analysis using SPSS program. Doxorubicin was used as a positive control in this assay. The test was conducted in triplicate.

\section{Cell Apoptosis Induction Test Using Flowcytometry Method}

The next step was testing the cell apoptosis induction of the most potential prepared compound (compound $\mathbf{5}$ and $\mathbf{8}$ ) using a flowcytometer. This procedure refered to Roihatul et al. ${ }^{18}$

\section{Computational Docking}

The docking experiment was carried out on Toshiba Satellite Pro C640, Intel(R) Core(TM) i3-2330M CPU @ 2.20 GHz, 4.00 GB memory (RAM) 32-bit system type, Windows 7 Ultimate Operation System. Docking experiment was performed using AutodockTools and Autodock4. Program ChemDraw Ultra 
RASĀYAN J. Chem.

Vol. 13 | No. 1 |654 - 662| January - March | 2020

12.0 was used to draw ligand. Ligand optimation was carried out using Program HyperChem 8.0.8 and UCSF Chimera 1.11.2. Program PyMOL 1.3, UCSF Chimera 1.11.2 and LigPlot+ v.2.1 were used to analyze the docking results. The 3D structure of p53 and MDM2 protein was taken from Protein Data Bank (PDB) with the access code 1YCR.

\section{Organic Synthesis}

\section{RESULTS AND DISCUSSION}

The synthesized compounds were obtained according to Claisen-Schmidt condensation as presented in Fig.-1. The IR spectra of compound 1-8 displayed a vibration band at $1691-1620 \mathrm{~cm}^{-1}$ corresponding to conjugated carbonyl which gave a signal at $\delta$ 193.6-194.8 ppm in ${ }^{13} \mathrm{C}$ NMR. ${ }^{1} \mathrm{H}$ NMR spectra of compound 1-8 showed a double signal with $J=15.4 \mathrm{~Hz}$ at 8.22-7.14 ppm for trans ethylenic group.

\section{Cytotoxic Assay}

To evaluate the cytotoxic activity, synthesized compounds were incubated with cervical cancer cells $(\mathrm{HeLa})$. The result of cytotoxicity assay $\left(\mathrm{IC}_{50}\right)$ is presented in Table-2.

Table-2: The Result of Cytotoxicity Assay using MTT Method

\begin{tabular}{c|c|c}
\hline Compound's Number & Compounds & $\mathrm{IC}_{50}(\mu \mathrm{M})$ \\
\hline 1 & 2-methoxy-2'- hydroxychalcone & 0.052 \\
\hline 2 & 3-methoxy-2'- hydroxychalcone & 0,029 \\
\hline 3 & 4-methoxy-2'- hydroxychalcone & 0,050 \\
\hline 4 & 2,4-dimethoxy-2'- hydroxychalcone & 0,074 \\
\hline 5 & 2,5- dimethoxy-2'- hydroxychalcone & 0,015 \\
\hline 6 & 4-N,N-dimethyl-2'- hydroxychalcone & 62,667 \\
\hline 7 & 4-fluoro-2'- hydroxychalcone & 0,054 \\
\hline 8 & 4-chloro-2'- hydroxychalcone & 0,016 \\
\hline 9 & Doxorubicin & 0,002 \\
\hline
\end{tabular}

These results showed that there are two compounds possessing potential $\mathrm{IC}_{50}$ that are 2,5-dimethoxy-2'hydroxychalcone $(0.015 \mu \mathrm{M})$ and 4-chloro-2'-hydroxychalcone $(0.016 \mu \mathrm{M})$. All the prepared chalcones exhibited lower activity compared to doxorubicin as positive control.

The apoptosis effect of the two most active prepared chalcones (2,5-dimethoxy-2'-hydroxychalcone and 4-chloro-2'-hydroxychalcone) against HeLa cancer cells line was investigated with a methode of double staining conjugated with Annexin V and Propidium iodide. The result of flow cytometry is presented in Fig.-2 and Table-3 respectively.

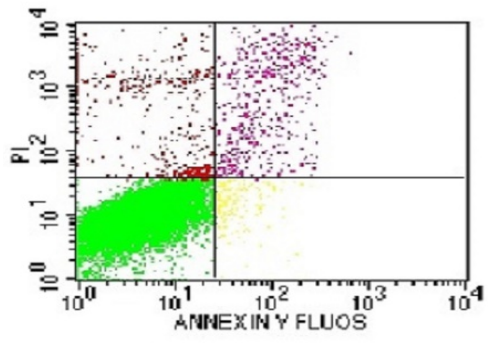

Control (HeLa cell without active compound)

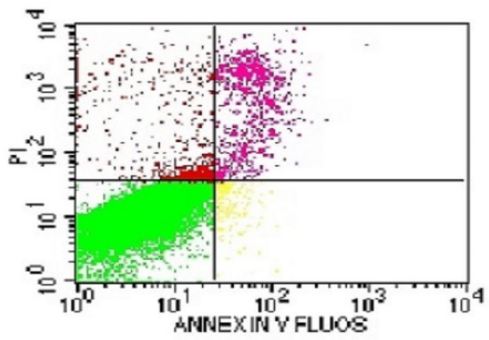

HeLa cell + $\mathrm{IC}_{50}$ of 4-chloro-2'hydroxychalcone

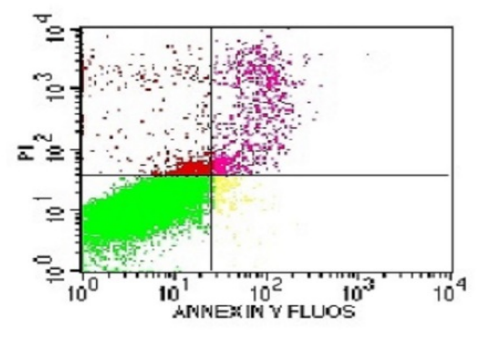

HeLa cell + IC 50 of 2,5-dimethoxy2 -hidroxychalcone

Fig.-2: The Result of Flow Cytometry Test (a) Control (HeLa Cell without Active Compound), (b) HeLa cell $+\mathrm{IC}_{50}$ of 4-chloro-2'-hydroxychalcone, (c)HeLa Cell $+\mathrm{IC}_{50}$ of 2,5-dimethoxy-2'-hydroxychalcone

From the Fig.-2 and Table-3, it can be seen that the treatment of the tested compound at $\mathrm{IC}_{50}$ concentrations on HeLa cells (24-hour incubation) did not provide significant results compared with control. The treatment using 4-chloro-2'-hydroxychalcone and 2,5-dimethoxy-2'-hydroxychalcone caused $5.04 \%$ and $4.47 \%$ respectively HeLa cells undergo late apoptosis (control $2.75 \%$ ). The treatment also 
RASĀYAN J. Chem.

Vol. 13 | No. 1 |654 - 662 | January - March | 2020

caused successively $4.75 \%$ and $4.56 \%$ of HeLa cells to experience necrosis (control $2.92 \%$ ). The results listed in Table 3 illustrate that the synthesized chalcone derivatives did not show the potential apoptotis activity although based on MTT test results, two chalcones (2,5-dimethoxy-2'-hydroxychalcone and 4chloro-2'-hydroxychalcone) showed very potential anticancer activity.

Table-3: The Result of Flow Cytometry Analysis of the Synthesized Compounds (5 and $\mathbf{8}$ )

\begin{tabular}{|c|c|c|c|c|}
\hline \multirow[b]{2}{*}{ Treatment on HeLa Cell } & \multicolumn{4}{|c|}{ Cell Population (\%) } \\
\hline & Viable Cells & $\begin{array}{c}\text { Early } \\
\text { Apoptosis }\end{array}$ & $\begin{array}{c}\text { Late } \\
\text { Apoptosis }\end{array}$ & Necrosis \\
\hline Control & 93.56 & 0.85 & 2.75 & 2.92 \\
\hline $\mathrm{IC}_{50}$ 4-chloro-2'-hydroxychalcone (8) & 89.48 & 0.90 & 5.04 & 4.75 \\
\hline $\mathrm{IC}_{50} 2,5$-dimethoxy-2'-hydroxychalcone (5) & 90.30 & 0.83 & 4.47 & 4.56 \\
\hline
\end{tabular}

The cell cycle inhibition that occurs could be observed by comparing the effects of the treatment with the synthesized compounds and control. The results of observations are listed in the Table- 4 . Based on the data obtained, it appears that there is no significant difference in results between tested compound and control. Even though the data obtained did not show high activity, it appeared that the inhibition of the HeLa cell cycle began at the G0-G1 phase, followed by the S phase and G2-M phase. 4-chloro-2'hydroxychalcone (8) showed slightly better activity than 2,5-dimethoxy-2'-hydroxychalcone (5).

Table-4: Analysis of HeLa Cells Cycle Treated with Synthesized Compounds (5 and 8)

\begin{tabular}{l|c|c|c|c|c}
\hline \multirow{2}{*}{ Treatment } & \multicolumn{5}{c}{ Cell Population (\%) } \\
\cline { 2 - 6 } & $\mathrm{M}_{1}$ & $\mathrm{G}_{0}-\mathrm{G}_{1}$ & $\mathrm{~S}$ & $\mathrm{G}_{2}-\mathrm{M}$ & $\mathrm{M}_{5}$ \\
\hline Control & 1.99 & 57.92 & 7.99 & 22.56 & 10.12 \\
\hline $\mathrm{IC}_{50}$ of 4-chloro-2'-hydroxychalcone (8) & 2.96 & 56.46 & 10.32 & 20.91 & 10.29 \\
\hline $\mathrm{IC}_{50}$ of 2,5-dimetoxy-2'-hydroxychalcone (5) & 2.67 & 56.48 & 9.23 & 20.82 & 11.52 \\
\hline
\end{tabular}

\section{Docking Molecular}

p53 is a tumor suppressor protein. As it is the human genome, protein p53 is a factor of transcription controlling the cellular response of cells to DNA damage through induction of cell-cycle cessation, reparation of DNA, apoptosis, or senescence. The p53 gene is deactivated in numerous human tumors, otherwise in cancer case it occurs an overexpression of MDM2. Therefore the disrupted MDM2-p53 interaction lead to greater levels of $\mathrm{p} 53$ and restoration of $\mathrm{p} 53$ transcriptional activity. ${ }^{16}$

In the MDM2-p53 complex structure, 2 intermolecular hydrogen bonds were found. One is in the middle of Phe-19 backbone amide of p53 and the Gln-72 side chain of MDM2 at the cleft entrance. Another is among nitrogen indole Trp-23 in p53 with the MDM2 Leu-54 backbone carbonyl, which is deep inside the cleft. The MDM2 cleft is positioned along with 14 preserved amino acids (hydrophobic and aromatic), which create many van der Waals contacts with p53. These 14 amino acids are Met-50, Leu-54, Leu-57, Gly-58, Ile-61, Met-62, Tyr-67, His-73, Val-75, Phe-91, Val-93, His-96, Ile-99, and Tyr-100 ${ }^{19}$. Fig.-3 presents the MDM2-p53 interaction.

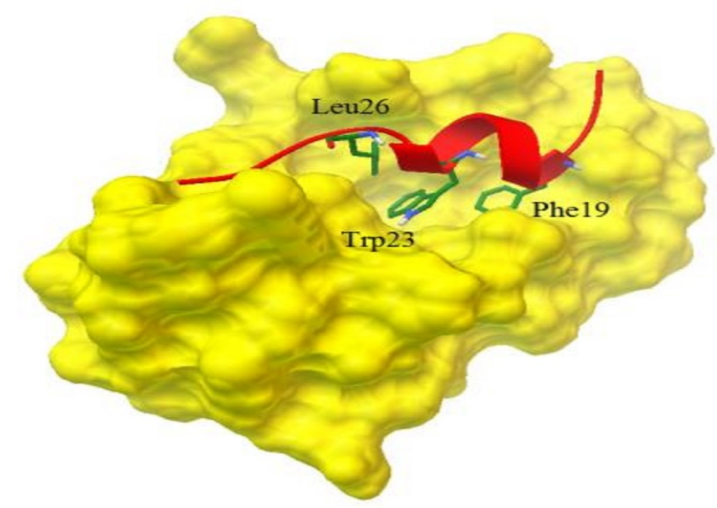

Fig.-3: Complex of MDM2 (Yellow) - p53 (Red)

659 
RASĀYAN J. Chem.

Vol. 13 | No. 1 |654 - 662| January - March | 2020

Docking molecular exhibited the interaction between synthesized compound as ligand and MDM2 protein target as shown in Table-5. This interaction was represented by $\Delta \mathrm{G}$ and $\mathrm{K}_{i .}{ }^{20}$ It can be seen in Table 5 that all chalcone compounds formed interactions with MDM-2. The hydrogen bond formed between chalcone and MDM2 mostly involved Leu-54 and His 96. Val-93 also formed interaction with one chalcone (Compound 1). Kussie et al had stated that two amino acids on MDM 2 which formed hydrogen bond with p53 were Gln-72 and Leu- $54 .{ }^{19}$ Therefore, the presence of chalcone which could form hydrogen bonds with Leu-54 on MDM2 will disrupt the MDM2-p53 interaction, as showed by compound 2, 3, 6, 7 and 8. The interaction between 4-chloro-2'-hydroxychalcone (8) and MDM2 showed the smallest $\Delta \mathrm{G}$ and $\mathrm{Ki}$, even though only one hydrogen bond was formed with an amino acid residue (Leu-54).

Table-5: Resume of Docking Results of the Prepared Compounds on MDM2

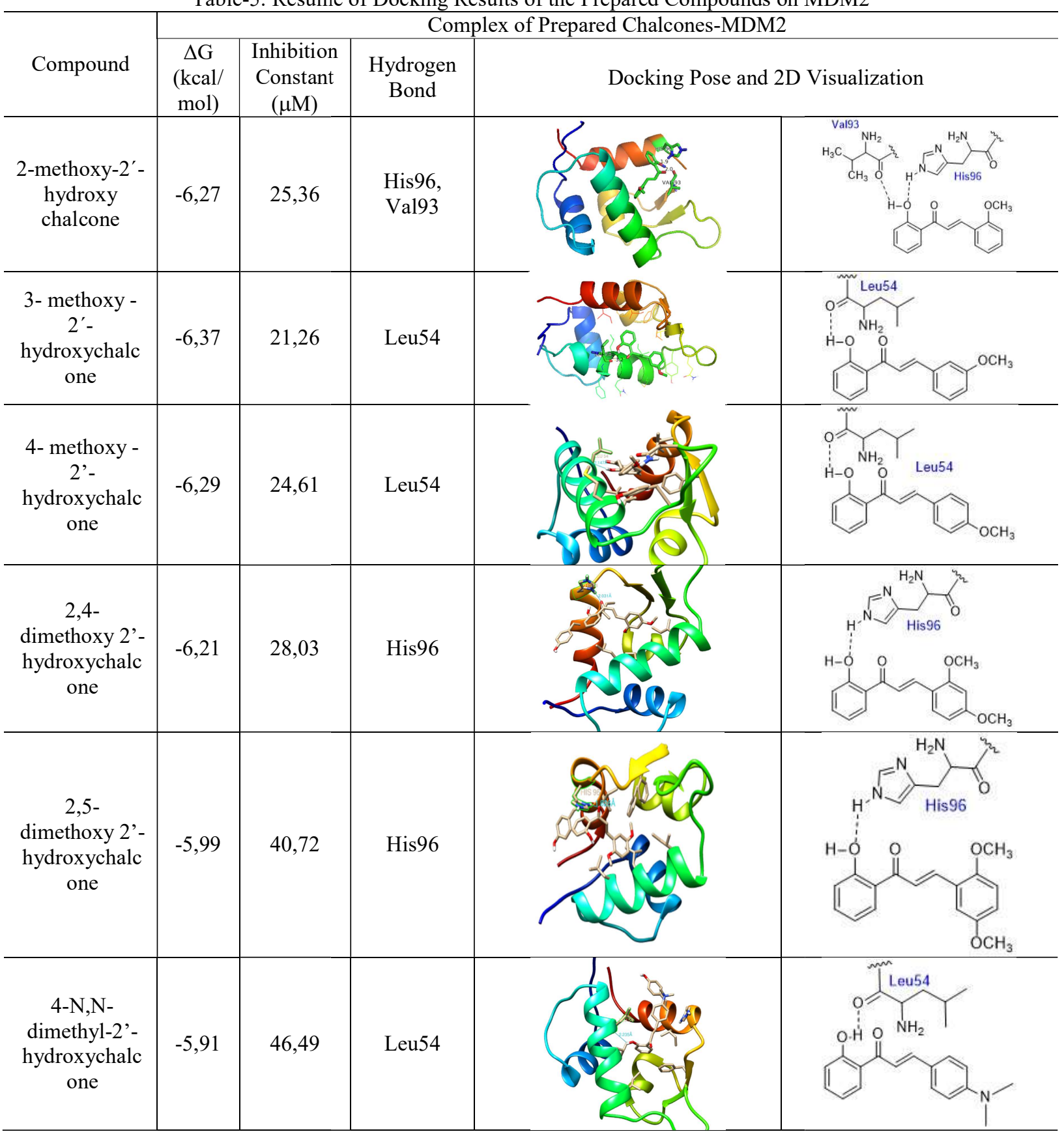


RASĀYAN J. Chem.

Vol. 13 | No. 1 |654 - 662| January - March | 2020

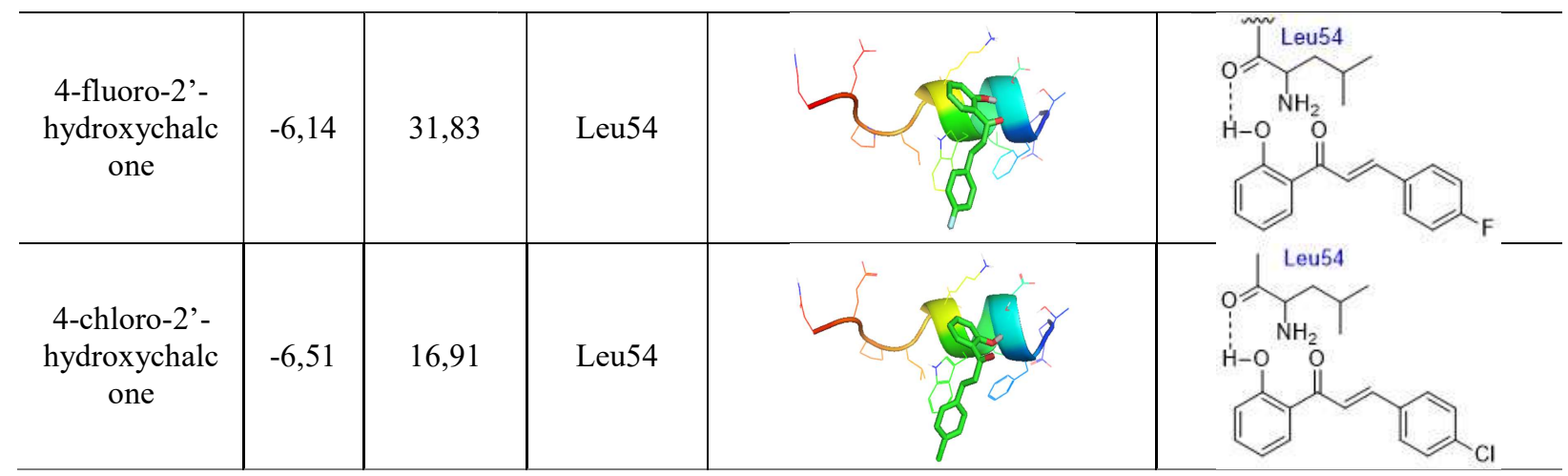

Visualization of molecular docking using Ligplot program also showed that beside hydrogen bond, 4chloro-2'- hydroxychalcone was also able to build van der Waals interaction with twelve amino acid residues at MDM2, that were Leu-54, Leu-57, Gly-58, Ile-61, Met-62, Tyr-67, Gln-72, Phe-86, Phe-91, Val-93, Ile-99, Ile-103 (Fig.-4).

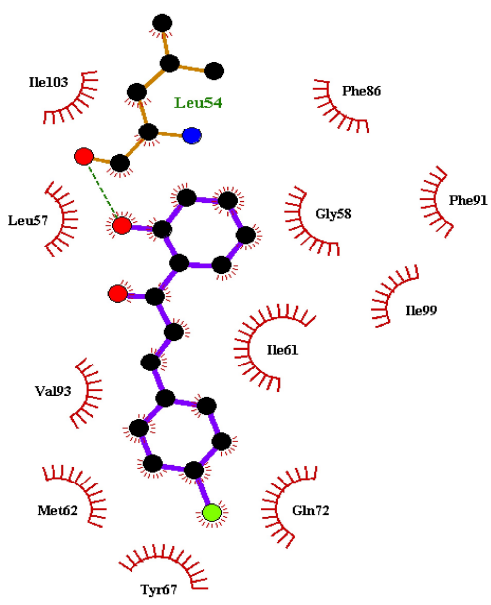

Fig.-4: Vander Waals Interaction between 4-Chloro 2'-hydroxycalkon with MDM2

Among the 12 amino acid residues in the MDM2 structure, there are 9 amino acid residues (hydrophobic and aromatic amino acids) which create many van der Waals contacts with p53. These 9 amino acids are Leu-54, Leu-57, Gly-58, Ile-61, Met-62, Tyr-67, Phe-91, Val-93, Ile-99. If the 9 amino acids form a van der Waals interaction with chalcone, then it can be ascertained that there will be interference with the interaction between MDM2-p53. So chalcone that could also form hydrophobic interactions with MDM2, in addition to hydrogen bonds, it will greatly disrupt in MDM2-p53 interaction.

The result of molecular docking was in accordance with the results of cytotoxicity test revealing that 4chloro-2'- hydroxychalcone showed the highest activity.

\section{CONCLUSION}

Eight 2'hydroxychalcones (1-8) derivatives compounds have been synthesized with 4-chloro-2'hydroxychalcone showing the best results in docking analysis while cytotoxicity test with MTT method exhibited that 2,5-dimethoxy-2'hydroxychalcone and 4-chloro-2'-hydroxychalcone gave very low $\mathrm{IC}_{50}$ of 0.15 and $0.16 \mu \mathrm{M}$ respectively, but both did not show potential apoptosis activity

\section{ACKNOWLEDGEMENT}

The research was funded by the Ministry of Research, Technology and Higher Education, of the Republic of Indonesia (Penelitian Dasar Unggulan Perguruan Tinggi, 2018). 
RASĀYAN J. Chem.

Vol. 13 | No. 1 |654 - 662| January - March | 2020

\section{REFERENCES}

1. M.M.H. Bhuiyan, M.I. Hossain, M.M. Mahmud, M. Al-Amin, Chemistry Journal, 1(1), 21(2011)

2. Y.S. Lee, S.S. Lim, K.H. Shin, Y.S. Kim, K. Ohuchi, S.H. Jung, Biological and Pharmaceutical Bulletin, 29(5), 1028(2006), DOI: 10.1248/bpb.29.1028

3. S. Vogel, M. Barbic, G. Juergenliemk, J. Heilmann, European Journal of Medicinal Chemistry, 45(6), 2206(2010), DOI:10.1016/j.ejmech.2010.01.060

4. G. Valdameri, C. Gauthier, R. Terreux, R. Kachadourian, B.J. Day, S.M.B. Winnischofer, M.E.M. Rocha, V. Frachet, X. Ronot, A. Di Pietro, A. Boumendjel, Journal of Medicinal Chemistry, 55(7), 3193(2012), DOI:10.1021/jm2016528

5. P. Boeck, C.A.B. Falcão, P.C. Leal, R.A. Yunes, V.C. Filho, E.C. Torres-Santos, B. RossiBergmann, Bioorganic and Medicinal Chemistry, 14(5), 1538(2006), DOI: $10.1016 /$ j.bmc.2005.10.005

6. T.D. Tran, T.T.N. Nguyen, T.H. Do, T.N.P. Huynh, C.D. Tran, K.M. Thai, Molecules, 17(6), 6684(2012), DOI: 10.3390/molecules 17066684

7. M. Liu, P. Wilairat, M.L. Go, Journal of Medicinal Chemistry, 44(25), 4443(2001), DOI: $10.1021 / \mathrm{jm} 0101747$

8. C.T. Hsieh, T.J. Hsieh, M. El-Shazly, D.W. Chuang, Y.H. Tsai, C.T. Yen, S.F. Wu, Y.C. Wu, F.R. Chang, Bioorganic and Medicinal Chemistry Letters, 22(12), 3912(2012), DOI: 10.1016/j.bmcl.2012.04.108

9. S.N.A. Bukhari, M. Jasamai, I. Jantan, W. Ahmad, Mini-Reviews in Organic Chemistry, 10(1), 73(2013), DOI:10.2174/1570193X11310010006

10. A.K. Babu, K. Selvaraju, Rasayan Journal of Chemistry, 11(4), 1501(2018), DOI: 10.31788/RJC.2018.1144037

11. M.R. Ahmed, V.G. Sastry, N. Bano, S. Ravichandra, M. Raghavendra, Rasayan Journal of Chemistry, 4(2), 289(2011)

12. A. Zamri, H.Y. Teruna, I. Ikhtiarudin, Molekul, 11(2), 299(2016), DOI: 10.20884/1.jm.2016.11.2.220

13. A.A.T. Suma, T.D. Wahyuningsih, Mustofa, Rasayan Journal of Chemistry, 12(2), 502(2019), DOI: $10.31788 /$ RJC.2019.1225020

14. Y. Haupt, R. Maya, A. Kazaz, M. Oren, Nature, 387, 296(1997), DOI:10.1038/387296a0

15. M.H.G. Kubbutat, S.N. Jones, K.H. Vousden, Nature, 387, 299(1997), DOI:10.1038/387299a0

16. R. Stoll, C. Renner, S. Hansen, S. Palme, C. Klein, A. Belling, W. Zeslawski, M. Kamionka, T. Rehm, P. Mühlhahn, R. Schumacher, F. Hesse, B. Kaluza, W. Voelter, R.A. Engh, T.A. Holak, Biochemistry, 40(2), 336(2001), DOI:10.1021/bi000930v

17. H. Suwito, Jumina, Mustofa, P. Pudjiastuti, M.Z. Fanani, Y. Kimata-Ariga, R. Katahira, T. Kawakami, T. Fujiwara, T. Hase, H.M. Sirat, N.N.T. Puspaningsih, Molecules, 19(12), 21473(2014), DOI:10.3390/molecules191221473

18. R. Mutiah, C. Indradmojo, H.D. Hardiyanti, T.P. Griana, A. Listyana, R Ramdhani, Journal of Applied Pharmaceutical Science, 7(10), 138(2017), DOI:10.7324/JAPS.2017.71020

19. P.H. Kussie, S. Gorina, V. Marechal, B. Elenbaas, J. Moreau, A.J. Levine, N.P. Pavletich, Science, 274(5289), 948(1996), DOI:10.1126/science.274.5289.948

20. R. Kim, J. Skolnick, Journal of Computational Chemistry, 29(8), 1316(2008), DOI: $10.1002 / j c c .20893$

[RJC-5534/2019] 